\title{
TECHNOLOGICAL MEASURES OF FOREFRONT ROAD IDENTIFICATION FOR VEHICLE COMFORT AND SAFETY IMPROVEMENT
}

\author{
Vidas ŽURAULIS ${ }^{1 *}$, Vytenis SURBLYS ${ }^{2}$, Eldar ŠABANOVIČ 3 \\ ${ }^{1,3}$ Transport and Logistics Competence Centre, Vilnius Gediminas Technical University, Lithuania \\ ${ }^{2}$ Dept of Automobile Engineering, Vilnius Gediminas Technical University, Lithuania
}

Received 29 January 2019; revised 10 April 2019; accepted 28 April 2019

\begin{abstract}
This paper presents the technological measures currently being developed at institutes and vehicle research centres dealing with forefront road identification. In this case, road identification corresponds with the surface irregularities and road surface type, which are evaluated by laser scanning and image analysis. Real-time adaptation, adaptation in advance and system external informing are stated as sequential generations of vehicle suspension and active braking systems where road identification is significantly important. Active and semi-active suspensions with their adaptation technologies for comfort and road holding characteristics are analysed. Also, an active braking system such as Anti-lock Braking System (ABS) and Autonomous Emergency Braking (AEB) have been considered as very sensitive to the road friction state. Artificial intelligence methods of deep learning have been presented as a promising image analysis method for classification of 12 different road surface types. Concluding the achieved benefit of road identification for traffic safety improvement is presented with reference to analysed research reports and assumptions made after the initial evaluation.
\end{abstract}

Keywords: road identification, road irregularities, laser scanning, semi-active suspension, damper, image analysis, friction state, deep learning, artificial intelligence, vehicle.

\section{Introduction}

Vehicle safety and comfort properties highly depend on the road where the vehicle is moving. From the perspective of its interaction with the wheel, the road, in respect of its design parameters, may be characterized by the type of the pavement and its smoothness. The smoothness of a road varies in course of its use. The natural environment and meteorological conditions cause changes in road condition (most frequently, in terms of cohesion and resistance to movement) and the long-lasting quality.

The modern society (in terms of civilization development) is not prone to change its habits or plans because of the environmental conditions. In terms of road traffic, it should be understood as a permanent satisfaction of the need in mobility independently on the environmental conditions the maximum possible comfort and safety. Vehicle manufacturers try to satisfy the said need by offering increasingly advanced technological systems to the market.

Upcoming technologies of autonomous vehicles require well-defined environmental perception (Tettamanti et al. 2016). However, weather conditions can influence the output data of various sensors that is why sensor fu- sion algorithms should be designed to evaluate environmental information.

In the paper, both technologies that are offered in the market already and those being developed by scientists and researchers are reviewed. Technologies of semi-active and active suspensions and active braking systems were chosen for the analysis because these systems are directly responsible for ensuring the comfort and safety of driving. In the most advanced systems, laser scanning of the forefront road surface and analysis of images fixed by a video camera on the base of artificial intellect are applied. In addition, communication technologies, like the ones of the not-to-distant future with a potential of broad application, are reviewed herein as well.

Section 1 of the paper is divided into three parts according to the technologies under analysis and presents a review of the existing and being developed vehicle systems worldwide. In Section 2, the research works carried out by the authors and the systems being created by them are presented. At the end of the paper, the conclusions and the possible benefit of the reviewed systems for improvement of traffic safety are formulated.

*Corresponding author. E-mail: vidas.zuraulis@vgtu.lt

\#Associate Editor of the TRANSPORT - the manuscript was handled by one of the Editors, who made all decisions related to the manuscript (including the choice of referees and the ultimate decision on the revision and publishing).

Copyright (C 2019 The Author(s). Published by VGTU Press

This is an Open Access article distributed under the terms of the Creative Commons Attribution License (http://creativecommons.org/licenses/by/4.0/), which permits unrestricted use, distribution, and reproduction in any medium, provided the original author and source are credited. 


\section{Review}

In this section, the advanced vehicle technologies are divided into three groups (Figure 1). For this purpose, the adaptation level according to the road properties identified upon applying different methods was chosen.

\subsection{System real-time adaptation}

Operation of systems capable to adapt themselves in real time is based on the correction of an operation mode of structural elements of the vehicle according to the parameters measured in real time. Such a system needs sensors and an Electronic Control Unit (ECU) that transforms the signals received from the sensors into the control signals (according to a mathematical algorithm). The sensors of this type systems measure the instantaneous parameters related to the operation of the same system. Such a principle of operation shows the main limitations: the system adapts itself according to the internal parameters of operation and with a delay. In addition, this principle of operation is universal enough, but never ensures the best possible result upon specific conditions.

In vehicles, passive suspensions are most widely used; however, vehicle manufacturers increasingly use semi-active and active suspensions. Such suspensions ensure the driving comfort, positively impact the interaction between the wheel and the road; in addition, they improve safety on the vehicle on its braking.

In literature, a semi-active suspension is analysed very frequently (Figure 2). The research works in this area are divided into two principal groups:

- characteristics of the damping element;

- control of a semi-active suspension.

A considerable share of scientists worldwide is involved in the investigation of these groups of semi-active suspensions (Savaresi, Spelta 2009; Savaresi et al. 2010; Mulla, Unaune 2013; Pei et al. 2016; Rao et al. 2010; Krauze, Kasprzyk 2016; Emam, Abdel Ghany 2012; Marzbanrad et al. 2013; Kashem et al. 2015; Aly, Salem 2013; Pepe, Carcaterra 2016; ZF Friedrichshafen AG 2011; Dąbrowski, Ślaski 2016).

A damping force in the dampers of a semi-active suspension may be changed in two ways: by increasing or decreasing the hole for liquid flowing in the damper, or; by varying liquid flow resistance (Wong 2008). In a semiactive system, two acceleration sensors are used: the first sensor is fixed to the unsprung mass (usually to the wheel hub or to the damper itself), the second sensor is arranged in the sprung mass, i.e. in the body of the vehicle. For each wheel of a vehicle, individual acceleration sensors are installed; however, sometimes only one body acceleration sensor is used for rear axle (ZF Friedrichshafen AG 2011). In addition, a system with the only acceleration sensor is being investigated as well (Savaresi, Spelta 2009). According to the obtained values of vertical accelerations, the control unit processes the data and sends a signal to the damper for changing its damping characteristics.

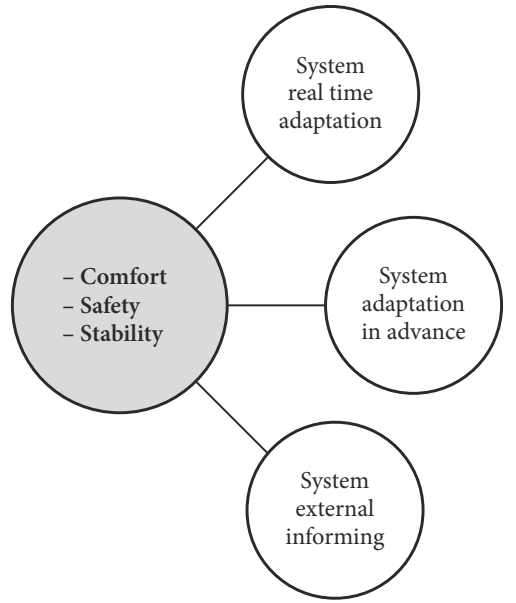

Figure 1. Groups of adaptation technologies for modern vehicle control

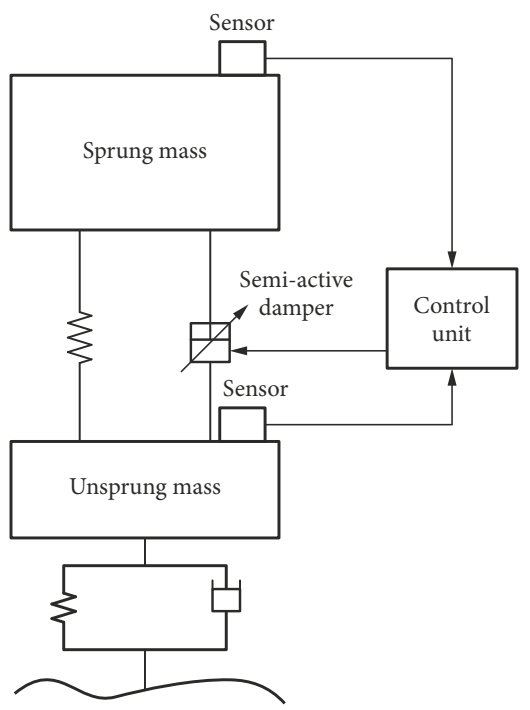

Figure 2. A semi-active suspension scheme (Wong 2008)

An active suspension differs from a semi-active suspension in its ability to change both damping and stiffness (Mulla, Unaune 2013). Both types of suspension use acceleration sensors to measure the accelerations and the shifts of the sprung and unsprung mass. In an active suspension, a considerable energy input (about $5 \ldots 10 \mathrm{~kW}$ ) is required for changing the damping and stiffness, whereas in a semiactive suspension $-10 \ldots 20 \mathrm{~W}$ only (Savaresi et al. 2010).

Beyond adaptive suspensions, other vehicle systems, such as braking or traction control, shall be also adjusted upon considering the conditions required to ensure the safety of driving. Vehicle Anti-lock Braking System (ABS) is being permanently improved to ensure the best possible stability of the vehicle during intensive braking. Sliding mode control (Sánchez-Torres et al. 2011), adaptive sliding - mode control (Zhang et al. 2014), a different number of phases in ABS logic (Bauer 1999; Gerard et al. 2010), artificial neural network (Eneh, Okafor 2014) or other (Aly et al. 2011) technologies of improvement are usable to adapt braking force control upon considering the exist- 
ing road conditions. Control strategies include identification of the critical wheel's slip ratio, angular acceleration and it's adapting to the existing road friction conditions. Although many algorithms are being improved upon striving for improving the braking efficiency on various road conditions, an optimum slip ratio in real time is being explored for improving lateral stability as well (Dinçmen et al. 2010). Nevertheless, the most important task is accurate enough identification or the road conditions as soon as possible (Zheng et al. 2011); however, in the case of adaptation according to the parameters of a system operating in real time, a delay takes place already, because the system will adapt itself only after a certain time of operation upon specific road conditions. An assessment of the road irregularities and ABS control adaptation are also important in cases of emergency braking on specific pavements (Ivanov et al. 2015; Van der Merwe et al. 2018; Žuraulis et al. 2018).

The other group of active vehicle braking systems includes Adaptive Cruise Control (ACC) and Autonomous Emergency Braking (AEB) systems. In most vehicles, ACC systems perform the longitudinal dynamics control function where the situation is identified by radars, cameras or other obstacle observance sensors and the braking system is activated automatically. Early generations of such systems only slowed down the vehicle or completely stopped it from the limited velocity $(30,50$ or $70 \mathrm{~km} / \mathrm{h})$. The appeared AEB systems intervened at a limited velocity as well; however, in course of improvement of the technologies, the systems became more sensitive, were activated at a higher initial velocity and ensured a higher intensity of braking that equalled or even exceeded the actions of the driver. In cases of intensive braking, the efficiency of braking highly depends on the road conditions - the type and the state of the pavement (Yi et al. 2002). On the base of the distance to the obstacle and velocity of its approaching, the supplemental AEB system establishes the critical moment of the situation and intervenes automatically by intensive braking of the vehicle. In such a case, the road conditions are of great importance for deciding on the critical moment, because the minimum safe distance to another vehicle, for example, on dry asphalt or snowy asphalt differs considerably (Koglbauer et al. 2018). In such a case, an adaptation of the braking system in real time according to the internal parameters of operation is insufficient and the system adaptation in advance is required. In this case, the critical braking moment shall be established both according to the position of the approaching obstacle and the real road conditions.

\subsection{System adaptation in advance}

The system adaptation in advance is mode advantaged, as compared to the system adaptation in real time. It's architecture is based by preparation of the system in advance for future conditions. In this case, the algorithm receive the information required for adaptation in advance from the additional sensors and have at least the minimum time for the preparation. Here sensors of other type are used because the distance of the contrary direction is measured, so video cameras or laser scanning are used. Such systems of sensors can establish a higher number of parameters of the forefront road; however, in many cases, the sensors or the processing of the information fixed are considerably more complicated, as compared to the above-discussed systems operating in real time.

In literature, various principles of suspension control are discussed upon. The principle based on an assessment of the irregularities of the road surface prior to starting of movement of the vehicle on the surface is frequently referred to as "preview control" (Dąbrowski, Ślaski 2016; Çalışkan et al. 2016). Usually, this principle of control is applied for an active suspension and is used for improving the driving comfort (Nwokah, Hurmuzlu 2001). Göhrle et al. (2015) used a laser sensor fixed to the windscreen: it measured the road irregularities prior to starting of movement of the vehicle on the surface under measurement. The obtained signal was used for improving the operation of the active suspension dampers. The authors faced problems related to vibrations of the sprung mass of the vehicle. While driving on irregularities of the road, the body of the vehicle vibrates together with the sensor fixed to the body. The other problem is filtering the signals received from the acceleration sensors and the gyroscope because the measured signals are very noisy.

The delayed operation of the dampers affects the principles of control that change the damping parameters in real time. The desirable values are reached after a certain period of time, not at the moment when they are required. One of the most important advantages of "preview control" principle of suspension control is the gained time for alteration of damping values of the damper.

Delays of a damper are divided into two principal groups (Więckowski et al. 2018):

- a delay of a generated and transmitted electric pulse signal from the control module to the valve of the dampers and its activation;

- a delay of alteration of the damping values of the damper.

Electric delay lasts for about $18 \mathrm{~ms}$, and hydromechanical delay (in the damper) - for about 55 ms (Figure 3). An alteration of damping values differs, if the damper operates in compression and rebound modes. During compression, a higher stiffness force of the spring resists, so it is calculated that the alteration takes about $67 \mathrm{~ms}$. When the damper operates in the rebound mode, the damping force values are altered more rapidly - for about $43 \mathrm{~ms}$ (Więckowski et al. 2018).

When the current strength starts changing, the damping force value starts changing as well. It is not necessary to wait for the moment when the current strength reaches the desirable value, so it is accepted that the total delay of the damper from the minimum lasts for about $0.055 \mathrm{~s}$ (Savaresi, Spelta 2009, Więckowski et al. 2018). 
a)

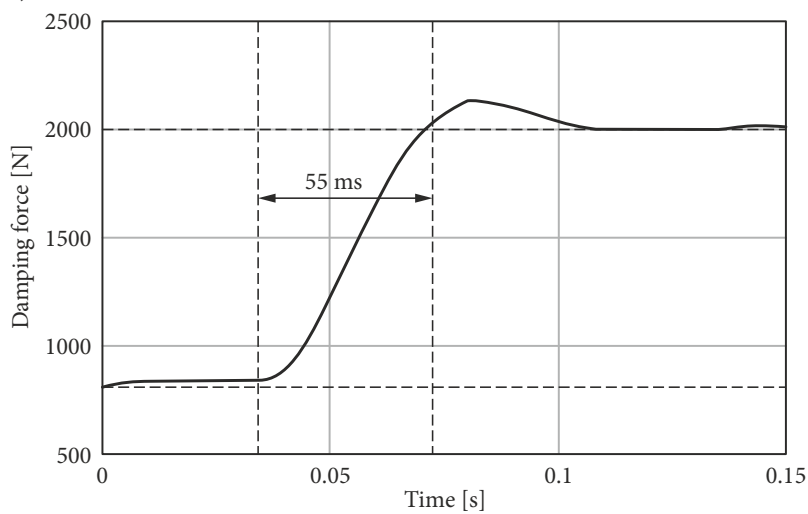

b)

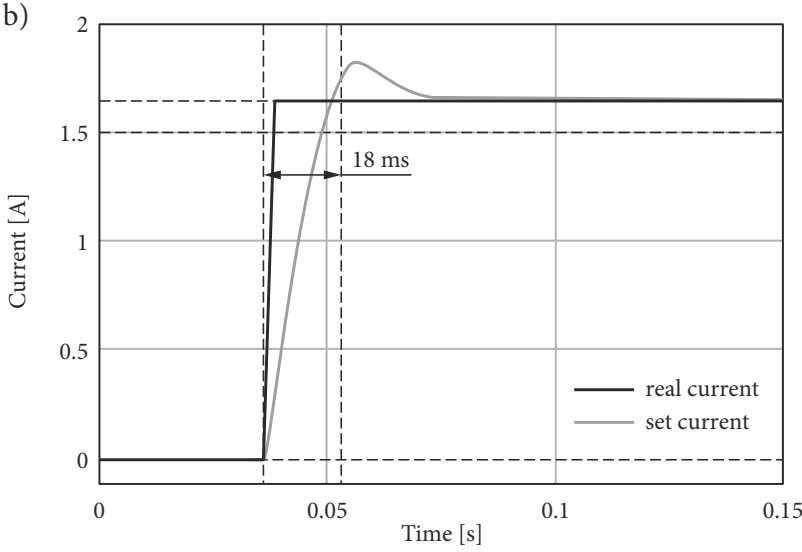

Figure 3. Damping force value and current time dependencies: $\mathrm{a}$ - damping force value; $\mathrm{b}$ - current (Więckowski et al. 2018)

The other fast developing area of forefront road identification is based on image processing from monocular and binocular (stereo) cameras for using the extracted data in Advanced Driving Assistance Systems (ADAS) (Krasner, Katz 2016; Prashanth et al. 2014) or self-driving vehicle systems (Yang et al. 2018; Mahmud et al. 2012; Milz et al. 2018). Monocular vision is usually used for determining weather and illumination (Gimonet et al. 2015; Cheng et al. 2018), path and obstacle (Nadav, Katz 2016), road, line, road edge detection and recognition (Yang et al. 2018; Van Hamme et al. 2013; Zhang, Wu 2009). Binocular vision can be efficiently used for object ranging, and usually shows better performance than monocular vision, especially in the task for creation of depth maps and point clouds from visual data, achieving results comparable or better than Light Detection And Ranging (LiDAR) (Smolyanskiy et al. 2018).

Most image processing can be done using histograms, thresholding and other traditional image processing methods (Oliveira, Correia 2008), but currently emerging trend is the usage of Deep Neural Network (DNN) based methods for feature extraction, image matching and decision making (Smolyanskiy et al. 2018; Meignen et al. 1997). Huge speed improvements are shown using Graphics Processing Unit (GPU) processing in embedded systems such as Jetson TX2 (Smolyanskiy et al. 2018). Availability of benchmark datasets such as Karlsruhe Institute of Technology and Toyota Technological Institute (KITTI) by Menze and Geiger (2015), Udacity (2016) promotes the development of even better processing methods for various use cases, but also there are research on simulated/ synthetic data (Gimonet et al. 2015) and newly collected datasets such as Shanghai Automotive Industry Corporation (SAIC) (Yang et al. 2018).

DNNs are susceptible to various physical-world attacks, such as false objects, like signs, or perturbations, that can cause misclassification and incorrect recognition (Eykholt et al. 2018), therefore development of DNNmodels that are not sensitive to image perturbations and learning more about correct positions of true objects on road is needed, that is the subject of functional safety (Rao, Frtunikj 2018). DNNs can be used for weather and illumination (Cheng et al. 2018), road surface estimation visual data (Naguib et al. 2017). In addition, there are developments where visual methods are used for road distress, cracks and other road damage detection (Cafiso et al. 2016; Shen 2016; Kashem et al. 2015; Meignen et al. 1997). We have not found research that is specializing on forefront DNN-based road surface type and condition prediction, therefore we began research in that direction, read more in Section 2.2.

\subsection{System external informing}

A system informing from external data sources is the most effective method of adaptation of the vehicle for forefront road conditions. In this case, an exact matching of the obtained to the desired road fragment and the predicted time of driving on it is of great importance. For data collection and transmission, external stationary sources (such as road conditions measurement databases, measurement databases, meteorological stations and so on) or other vehicles may be used. So, the communication system of a vehicle (Vehicle-to-Everything - V2X) can be associated with the road infrastructure (Vehicle-to-Infrastructure - V2I), other vehicles (Vehicle-to-Vehicle - V2V) or a specialized data network (Vehicle-to-Network - V2N). An advantage of such a conception is sufficient time for adapting systems of the vehicle; however, insufficient reliability of transmission and a need for special communication equipment should be identified as imperfections of the system. Because of their broad applicability, these systems are rapidly developed in the sector of autonomous vehicles.

V2X systems are based on various communication technologies and may be classified according to the type of communication:

- the basic station - a communication centre (Virtualto-Physical - V2P; V2I);

- a communication centre (V2V);

- a network where communication centres are interconnected and transfer the data (mesh network) (V2V).

V2P systems are used to ensure a communication between the vehicle and pedestrians in order to warn the latter on the approaching vehicle, whereas a communication with the infrastructure (V2I) may be ensured by Ra- 
dio-Frequency IDentification (RFID) technology or lowpower transmission technology integrated in the surface of the road usable, for example, for observing the road edge or easier identification of the stop line (road signs) or by distant traffic information from the control system of city transport flows.

At present, it is often spoken that in future self-propelled vehicles, because of abundant sensors and large flows of data generated by them, should be provided with a communication system ensuring a higher data transmission velocity; the most attention is paid to $5 \mathrm{G}$ mobile communication technologies; however, it is emphasized that for transport systems, a special network (separated from the general-purpose network) should be formed because a lower delay, as well as high safety and reliability, are of a greater importance for it.

LTE Direct is the autonomous long-distance Device-to-Device (D2D) protocol introduced in 3GPP Release 12 specification (https://www.3gpp.org/specifications/ releases/68-release-12). This communication protocol will be used for direct communication between nearby LTE devices, therefore, measurement data from one vehicle will be available for other vehicles moving behind it that can use this data for comfort and safety systems adaptation in advance. In addition, other kinds of V2X can be used for providing data about forefront road conditions.

\section{Application cases for adaptation systems}

\subsection{Laser scanning preparation for suspension adaptation}

Measuring the distance to the road surface enables to predict its irregularities; however, a proper result is obtained after completion of the required corrections of the signal. A laser distance sensor is mounted on the sprung mass, so its output signal measures the vibrations appearing be- cause of stiffness and damping of the suspension as well as of inertia of the vehicle, not the real road surface. For processing a laser sensor signal, a compensation algorithm (Figure 4) had been developed (Surblys et al. 2018). The algorithm eliminates the following 3 parameters from the signal under measuring:

- a vertical shift of the sprung mass in the point of the centre of gravity;

- a vertical shift of the sprung mass in the laser sensor mounting position that is caused by the pitch oscillation;

- the laser sensor mounting position (for a sensor mounted at an angle).

For testing the developed algorithm, experimental tests had been carried out. During the tests, the vehicle was moving with a velocity of $30 \mathrm{~km} / \mathrm{h}$ on $0.05 \mathrm{~m}$ high and $0.35 \mathrm{~m}$ wide sinusoidal obstacle (traffic speed reduction element). Tests were carried out on the $60 \mathrm{~m}$ section. The road surface was a smooth paving. The following vehicle dynamic factors were recorded: body vertical acceleration, body pitch velocity and laser signal. Laser was mounted in front of vehicle, on the front bumper.

In Figure 5, the results of the tests with and without compensation are provided. The obtained curves show that the compensation algorithm reduces the vertical oscillations of the sprung mass and enables estimating the real road profile. The data were fixed on an acceleration of the vehicle as well, so the measured laser signal distinguished for oscillations of high amplitude at the beginning (Figure 5, the zone 1).

A real road profile is provided in Figure 6. The laser sensor had fixed a $0.067 \mathrm{~m}$ bump; after the compensation of the bump was $0.052 \mathrm{~m}$ (the real height of the bump is $0.05 \mathrm{~m}$ ). In addition, striking oscillations appeared after the vehicle wheels had overridden the obstacle were countervailed as well (Figure 5, the zone 2).

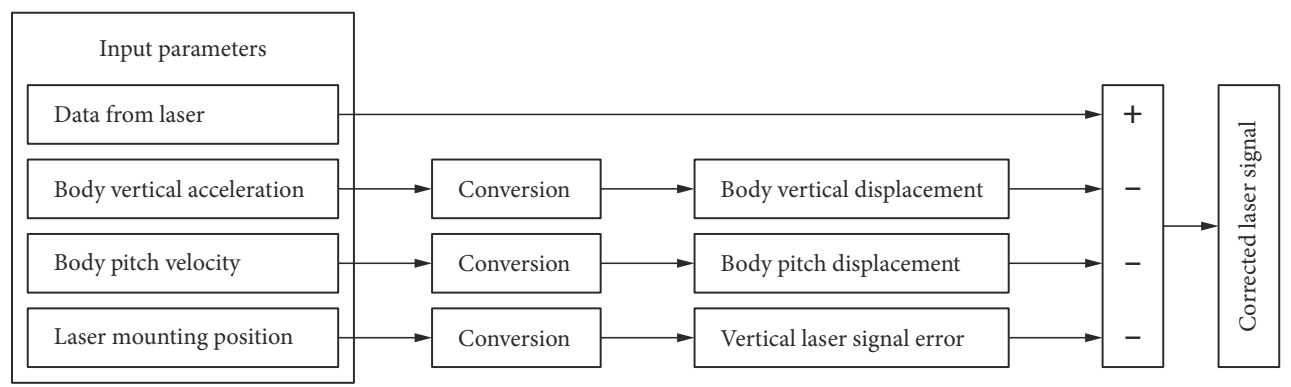

Figure 4. Laser sensor compensation for sprung mass dynamics (Surblys et al. 2018)

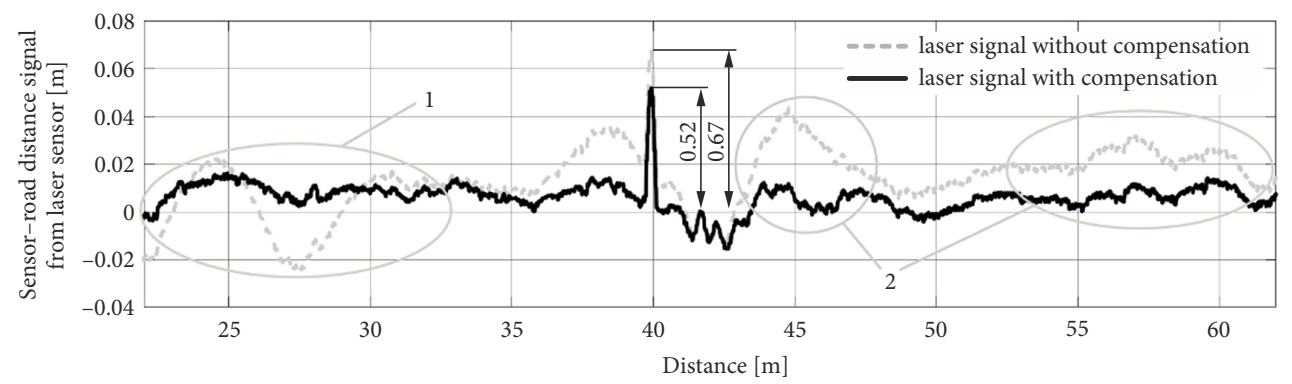

Figure 5. Estimating the road profile using a laser sensor when the suspension is set to minimum damping (Surblys et al. 2018) 


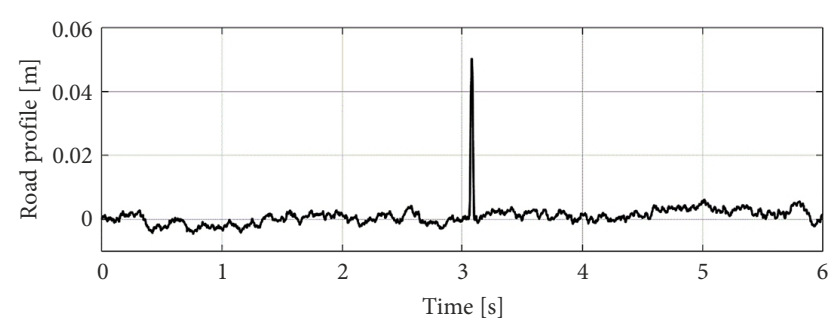

Figure 6. Road profile when velocity is $30 \mathrm{~km} / \mathrm{h}$

Laser-based measuring technologies present a sufficiently reliable tool for provision of information the geometrical properties of the approaching obstacle; however, a safe and appropriate application of sensors, precizity of their mounting and their prices are the issues of great importance as well.

\subsection{Image analysis for pavement state identification}

One of these research areas is image analysis for forefront road surface identification. Emerging artificial intelligence methods of deep learning were selected for classification of 12 different road surface types:

- ice;

- snow wet;

- snow dry;

- gravel (dirt) wet;

- gravel (dirt) dry;

- cobble wet;

- cobble dry;

- concrete wet;

- concrete dry;

- asphalt wet;

- asphalt moist;

- asphalt dry.

DNNs require big datasets to learn from. Therefore, more than 250 thousand labelled images for road surface type dataset were collected and labelled. A typical car camera was used to collect $1920 \times 1080$ pixels resolution video. This video was split into frames and frames taken during different seasons were collected. This way all kinds of weather conditions and road surfaces were gathered.

A DNN AlexNet with changes as input image size and several other parameters was used. The total structure of the network is presented in Table 1.

This DNN was made of 5 convolutional layers and 3 fully connected layers, all with Rectified Linear Units (ReLU) as the activation function. In order to reduce classifier dependence on separate pixels, the Dropout layers were used.

The dataset was formed by squeezing image frames into the $512 \times 512$ pixels size frame as input (Figure 7). This size was selected because $256 \times 256$ pixels size images had not enough details of the road texture. The DNN was trained on GPU workstation using CAFFE then converted into TensorRT and tested on $n$ Vidia Jetson TX2 AI platform. This platform consumes only up to $15 \mathrm{~W}$ of power and provides up to 1 TFLOP of DNN calculations. It provided real-time video processing of $30 \mathrm{fps}$, at $20 \mathrm{~ms}$
Table 1. Structure of DNN-based road type classifier

\begin{tabular}{|c|c|}
\hline Layer & Parameters \\
\hline Convolution 1 & $96 \times 11 \times 11$, stride 8 \\
\hline Activation 1 & ReLU \\
\hline Pooling 1 & MAX, $3 \times 3$, stride 2 \\
\hline Convolution 2 & $256 \times 5 \times 5$, stride 1 , groups 2 \\
\hline Activation 2 & ReLU \\
\hline Pooling 2 & MAX, $3 \times 3$, stride 2 \\
\hline Convolution 3 & $384 \times 3 \times 3$, stride 1 \\
\hline Activation 3 & ReLU \\
\hline Convolution 4 & $384 \times 3 \times 3$, stride 1 , groups 2 \\
\hline Activation 4 & ReLU \\
\hline Convolution 5 & $256 \times 3 \times 3$, stride 1 , groups 2 \\
\hline Activation 5 & ReLU \\
\hline Pooling 5 & MAX, $3 \times 3$, stride 2 \\
\hline Fully-connected 6 & 4096 \\
\hline Activation 6 & ReLU \\
\hline Dropout6 & Ratio of 0.5 \\
\hline Fully-connected 7 & 4096 \\
\hline Activation 7 & ReLU \\
\hline Dropout 7 & Ratio of 0.5 \\
\hline Fully-connected 8 & 12 \\
\hline Softmax & - \\
\hline
\end{tabular}

processing per frame, that would mean about half meter latency at speed of $100 \mathrm{~km} / \mathrm{h}$. The road types were classified with up to $97.35 \%$ accuracy on the validation set and $84 \%$ on the new data that was collected later. The accuracy and frequent classification mistakes made by the neural network were analysed and these aspects were found:

- both the dry and wet concrete classes were identified as the best with less than $1 \%$ error. Such a small error rate can be achieved due to small variation between training, evaluation and testing data, as there was only one such kind of road in the dataset;

- the dry, moist and wet asphalt detection were good, sometimes errors occurred due to dark conditions during the night or in tunnels. Also, some mistakes were found due to the variation of asphalt brightness and shadows under bridges;

- the dry and wet gravel detection was good, most errors occurred because there was an unclear boundary between the dry and wet gravel. Mostly the dry gravel is misclassified as the dry asphalt, and the wet gravel as the dry gravel;

- the dry and wet cobble road detection was worse because of the vibration effect on the camera image, the images of that road often are too blurry to get enough details, and usually, it can look similar to the dry asphalt or gravel. In addition, this class has the least amount of samples in the current dataset. The dry cobble is misclassified as the dry asphalt and the wet cobble, and the wet cobble as the dry asphalt or wet snow; 

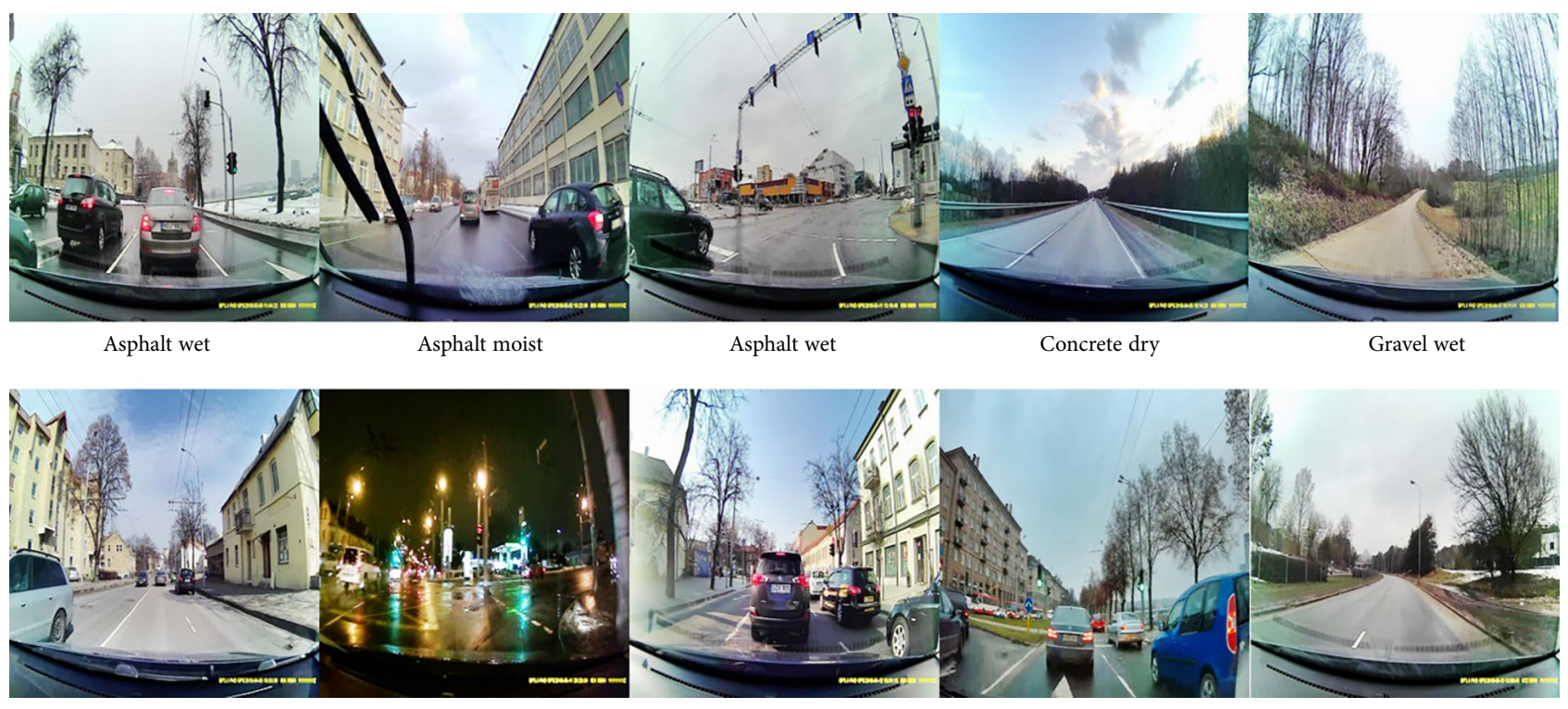

Asphalt dry

Asphalt wet

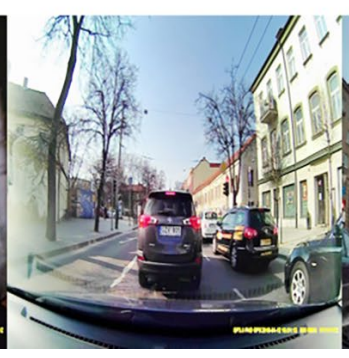

Asphalt dry

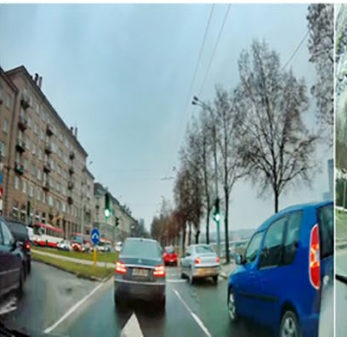

Asphalt moist



Asphalt wet

Figure 7. Example images from the collected dataset

- the dry and wet snow were identified satisfactory, there were cases in samples where only part of the road was covered in snow and other parts were asphalt, therefore some was misclassified as asphalt. It is planned to move such samples to asphalt samples;

- the ice detection was generally bad because even for a human it is hard to visually discern between ice and wet asphalt. Therefore, ice detection will be removed in future revisions.

Based on findings road type classification can be improved by the better quality camera and additional examples of some road surface types. Moreover, the assumption can be made, that accuracy can further be improved by detecting and extracting only road surface from the camera images. This way more surface information will be available in the same image size compared to the resized full image.

The output from this kind of system can be useful for Emergency braking, as from determined road state it can better predict Time To Collision (TTC) for its typical values of $0.8 \ldots 2.5 \mathrm{~s}$ (Koglbauer et al. 2018). Different kinds of AEB and ACC systems are designed to work in some range of velocities and full or partly braking is chosen. AEB effectiveness can be improved by forefront knowledge of driving conditions as this can also predict what road friction (coefficient $\mu$ ) can be expected in advance.

The data received from this system can be used for in advance prediction of ABS or Electronic Stability Program (ESP) systems settings selection. For example, with the help of forefront road sensing, system's thresholds variables for road friction (coefficient $\mu$ ), wheel angular accelerations $\mathrm{A},+\mathrm{a},-\mathrm{a}$, could be selected in advance and response time and effectiveness improved as result.

On identification of forefront road condition, difficulties most frequently are caused by non-uniform illumination and shadows. The dynamic range of a video sensor of the camera is limited and adjusting the image brightness requires a certain time; nevertheless, some details are lost in the darkest and brightest fragments of the image. In addition, if the image brightness varies, the task of identification becomes more complicated, because the developed algorithm should ensure resistance to changes in illumination. Shadows of viaducts and bridges, as well as dark parts of tunnels, provide major difficulties as well: they may be mistaken for wet pavement. Therefore, shadow compensation methods should be applied in this sector in future.

The other complicated case is poor visibility in case of a mist, heavy rain, hail or snowstorm - in such cases, visible light spectrum cameras, as a human vision, cannot ensure a good identification of the pavement; however, it is possible to identify the type of the weather.

\section{Conclusions}

All vehicles technology progress leads to improvements in advanced driver assistance systems and also partly or fully autonomous vehicles. Vehicle suspension, active braking systems increase driving comfort and safety, but a different level of systems adaptation to road conditions can significantly improve the desired vehicle performance. The information about road surface irregularities before vehicle cross it helps to eliminate electronic and hydromechanical delay, but vehicle body oscillations and sensor mounting should be taken into account before. Moreover, such kind of information can be effectively used in vehicle communication technologies to supplement road infrastructure database and to send direct messages for other vehicles.

As emerging artificial intelligence methods of deep learning can be successfully implemented for type of road surface classification, DNNs require big datasets to learn from. More than 250 thousand labelled images for road 
surface type dataset were collected and labelled for 12 different road surface types. Nevertheless, the method shows promising results as information about road type and its state expands the efficiency of vehicle active safety. As road sensing is based on image analysis, no complicated intervention to vehicle construction is required except typical camera mounted as video registrator inside the vehicle.

However, the main risks and limitations for further development include:

- response speed - methods for faster that human driver performance should be investigated to enable better comfort and safety;

- price - all new technologies are expensive and available only in luxury vehicles, that form a small part of all vehicles, therefore intelligent usage of cheaper sensors and processing should be developed;

- Artificial Intelligence (AI) learning data - AI requires a lot of data to be collected and prepared for training, therefore lots of time and resources are needed;

- cybersecurity - all complex connected systems are potentially vulnerable to private data theft and remote hijacking of vehicle control;

- unpredictable obstacles - there are a lot of rare situations, therefore safety systems that can prevent the system from unpredicted behaviour should be provided;

- user acceptance - it is important how these systems are marketed and if they truly are easy to use and useful for end-user;

- certification - certification is important for all automotive hardware and software, and there is a growing need to create certification methods for automotive systems based on artificial intelligence.

\section{References}

Aly, A. A.; Salem, F. A. 2013. Vehicle suspension systems control: a review, International Journal of Control, Automation and Systems 2(2): 46-54.

Aly, A. A.; Zeidan, E.-S.; Hamed, A.; Salem, F. 2011. An antilockbraking systems (ABS) control: a technical review, Intelligent Control and Automation 2(3): 186-195. https://doi.org/10.4236/ica.2011.23023

Bauer, H. (Ed.). 1999. Driving-Safety Systems. Society of Automotive Engineers (SAE). 250 p.

Cafiso, S.; D’Agostino, C.; Delfino, E.; Montella, A. 2016. From manual to automatic pavement distress detection and classification, in 2017 5th IEEE International Conference on Models and Technologies for Intelligent Transportation Systems (MT-ITS), 26-28 June 2017, Naples, Italy, 433-438. https://doi.org/10.1109/MTITS.2017.8005711

Çalışkan, K.; Henze, R.; Küçükay, F. 2016. Potential of road preview for suspension control under transient road inputs, IFAC-PapersOnLine 49(3): 117-122.

https://doi.org/10.1016/j.ifacol.2016.07.020

Cheng, G.; Zheng, J. Y.; Murase, H. 2018. Sparse coding of weather and illuminations for ADAS and autonomous driving, in 2018 IEEE Intelligent Vehicles Symposium (IV), 26-30 June 2018, Changshu, China, 2030-2035.

https://doi.org/10.1109/IVS.2018.8500385
Dąbrowski, K.; Ślaski, G. 2016. Method and algorithm of automatic estimation of road surface type for variable damping control, IOP Conference Series: Materials Science and Engineering 148: 012028.

https://doi.org/10.1088/1757-899X/148/1/012028

Dinçmen, E.; Acarman, T.; Aksun Güvenç, B. 2010. ABS control algorithm via extremum seeking method with enhanced lateral stability, IFAC Proceedings Volumes 43(7): 19-24. https://doi.org/10.3182/20100712-3-DE-2013.00017

Emam, A. S.; Abdel Ghany, A. M. 2012. Enhancement of ride quality of quarter vehicle model by using mixed $\mathrm{H} 2 / \mathrm{H}$ with pole-placement, Engineering 4(2): 126-132. https://doi.org/10.4236/eng.2012.42016

Eneh, I. I.; Okafor, P. U. 2014. Design of an automatic brake control system using artificial neural network, International Journal of Scientific \& Engineering Research 5(4): 1239-1245.

Eykholt, K.; Evtimov, I.; Fernandes, E.; Li, B.; Rahmati, A.; Xiao, C.; Prakash, A.; Kohno, T.; Song, D. 2018. Robust physical-world attacks on deep learning visual classification, in 2018 IEEE/CVF Conference on Computer Vision and Pattern Recognition, 18-23 June 2018, Salt Lake City, UT, USA, 1625-1634. https://doi.org/10.1109/CVPR.2018.00175

Gerard, M.; Pasillas-Lépine, W.; De Vries, E.; Verhaegen, M. 2010. Adaptation of hybrid five-phase ABS algorithms for experimental validation, IFAC Proceedings Volumes 43(7): 13-18. https://doi.org/10.3182/20100712-3-DE-2013.00021

Gimonet, N.; Cord, A.; Saint Pierre, G. 2015. How to predict real road state from vehicle embedded camera?, in 2015 IEEE Intelligent Vehicles Symposium (IV), 28 June-1 July 2015, Seoul, South Korea, 593-598. https://doi.org/10.1109/IVS.2015.7225749

Göhrle, C.; Schindler, A.; Wagner, A.; Sawodny, O. 2015. Road profile estimation and preview control for low-bandwidth active suspension systems, IEEE/ASME Transactions on Mechatronics 20(5): 2299-2310.

https://doi.org/10.1109/TMECH.2014.2375336

Ivanov, V.; Savitski, D.; Augsburg, K.; Barber, P.; Knauder, B.; Zehetner, J. 2015. Wheel slip control for all-wheel drive electric vehicle with compensation of road disturbances, Journal of Terramechanics 61: 1-10.

https://doi.org/10.1016/j.jterra.2015.06.005

Kashem, S. B. A.; Chowdhury, M. A.; Choudhury, T. A.; Ektesabi, M.; Nagarajah, R. 2015. Study and review on vehicle suspension control theories and introduction of novel adaptive skyhook control system, Australian Journal of Basic and Applied Sciences 9(30): 1-12.

Koglbauer, I.; Holzinger, J.; Eichberger, A.; Lex, C. 2018. Autonomous emergency braking systems adapted to snowy road conditions improve drivers' perceived safety and trust, Traffic Injury Prevention 19(3): 332-337.

https://doi.org/10.1080/15389588.2017.1407411

Krasner, G.; Katz, E. 2016. Automatic parking identification and vehicle guidance with road awareness, in 2016 IEEE International Conference on the Science of Electrical Engineering (ICSEE), 16-18 November 2016, Eilat, Israel, 1-5.

https://doi.org/10.1109/ICSEE.2016.7806133

Krauze, P.; Kasprzyk, J. 2016. Comparison of linear and nonlinear feedback control for a half-car model with MR dampers, in 2016 21st International Conference on Methods and Models in Automation and Robotics (MMAR), 29 August-1 September 2016, Miedzyzdroje, Poland, 965-970.

https://doi.org/10.1109/MMAR.2016.7575268 
Mahmud, F.; Arafat, A.; Zuhori, S. T. 2012. Intelligent autonomous vehicle navigated by using artificial neural network, in 2012 7th International Conference on Electrical and Computer Engineering, 20-22 December 2012, Dhaka, Bangladesh, 105108. https://doi.org/10.1109/ICECE.2012.6471496

Marzbanrad, J.; Poozesh, P.; Damroodi, M. 2013. Improving vehicle ride comfort using an active and semi-active controller in a half-car model, Journal of Vibration and Control 19(9): 1357-1377. https://doi.org/10.1177/1077546312441814

Meignen, D.; Bernadet, M.; Briand, H. 1997. One application of neural networks for detection of defects using video data bases: identification of road distresses, in Database and Expert Systems Applications. 8th International Conference, DEXA'97. Proceedings, 1-2 September 1997, Toulouse, France, 459-464. https://doi.org/10.1109/DEXA.1997.617332

Menze, M.; Geiger, A. 2015. Object scene flow for autonomous vehicles, in 2015 IEEE Conference on Computer Vision and Pattern Recognition (CVPR), 7-12 June 2015, Boston, MA, USA, 3061-3070. https://doi.org/10.1109/CVPR.2015.7298925

Milz, S.; Arbeiter, G.; Witt, C.; Abdallah, B.; Yogamani, S. 2018. Visual SLAM for automated driving: exploring the applications of deep learning, in 2018 IEEE/CVF Conference on Computer Vision and Pattern Recognition Workshops (CVPRW), 18-22 June 2018, Salt Lake City, UT, USA, 360-369. https://doi.org/10.1109/CVPRW.2018.00062

Mulla, A. A.; Unaune, D. R. 2013. Active suspensions future trend of automotive suspensions, in International Conference on Emerging Trends in Technology \& its Applications (ICETTA-2013), 6-7 March 2013, Karjat Mumbai, India, 1-9.

Nadav, I.; Katz, E. 2016. Off-road path and obstacle detection using monocular camera, in 2016 IEEE International Conference on the Science of Electrical Engineering (ICSEE), 16-18 November 2016, Eilat, Israel, 1-5.

https://doi.org/10.1109/ICSEE.2016.7806132

Naguib, A. M.; Kim, J.; Lee, S. 2017. 3D environmental modeling and drivable road identification for a long-range rover, in 2017 14th International Conference on Ubiquitous Robots and Ambient Intelligence (URAI), 28 June-1 July 2017, Jeju, South Korea, 697-700. https://doi.org/10.1109/URAI.2017.7992802

Nwokah, O. D. I.; Hurmuzlu, Y. 2001. The Mechanical Systems Design Handbook: Modeling, Measurement, and Control. CRC Press. 872 p.

Oliveira, H.; Correia, P. L. 2008. Identifying and retrieving distress images from road pavement surveys, in 2008 15th IEEE International Conference on Image Processing, 12-15 October 2008, San Diego, CA, USA, 57-60.

https://doi.org/10.1109/ICIP.2008.4711690

Pei, Q.; Na, J.; Huang, Y.; Gao, G.; Wu, X. 2016. Adaptive estimation and control of MR damper for semi-active suspension systems, in 2016 35th Chinese Control Conference (CCC), 27-29 July 2016, Chengdu, China, 3111-3116. https://doi.org/10.1109/ChiCC.2016.7553836

Pepe, G.; Carcaterra, A. 2016. VFC - variational feedback controller and its application to semi-active suspensions, $\mathrm{Me}$ chanical Systems and Signal Processing 76-77: 72-92. https://doi.org/10.1016/j.ymssp.2016.01.002

Prashanth, C.; Mala, J.; Santhosh, K. S.; Mithilesh, N. S. R. 2014. Road tracking using particle filters for Advanced Driver Assistance Systems, in 17th International IEEE Conference on Intelligent Transportation Systems (ITSC), 8-11 October 2014, Qingdao, China, 1408-1414.

https://doi.org/10.1109/ITSC.2014.6957884
Rao, Q.; Frtunikj, J. 2018. Deep learning for self-driving cars: chances and challenges, in SEFAIS'18: Proceedings of the 1st International Workshop on Software Engineering for AI in Autonomous Systems, 28 May 2018, Gothenburg, Sweden, 35-38. https://doi.org/10.1145/3194085.3194087

Rao, T. R. M.; Rao, G. V.; Rao, K. S.; Purushottam, A. 2010. Analysis of passive and semi active controlled suspension systems for ride comfort in an omnibus passing over a speed bump, International Journal of Research and Reviews in Applied Sciences 5(1): 7-17.

Sánchez-Torres, J. D.; Loukianov, A. G.; Galicia, M. I.; Rivera, J. 2011. A sliding mode regulator for antilock brake system, IFAC Proceedings Volumes 44(1): 7187-7192.

https://doi.org/10.3182/20110828-6-IT-1002.03644

Savaresi, S.; Poussot-Vassal, C.; Spelta, C.; Sename, O.; Dugard, L. 2010. Semi-Active Suspension Control Design for Vehicles. Butterworth-Heinemann. 240 p.

Savaresi, S. M.; Spelta, C. 2009. A single-sensor control strategy for semi-active suspensions, IEEE Transactions on Control Systems Technology 17(1): 143-152.

https://doi.org/10.1109/TCST.2008.906313

Shen, G. 2016. Road crack detection based on video image processing, in 2016 3rd International Conference on Systems and Informatics (ICSAI), 19-21 November 2016, Shanghai, China, 912-917. https://doi.org/10.1109/ICSAI.2016.7811081

Smolyanskiy, N.; Kamenev, A.; Birchfield, S. 2018. On the importance of stereo for accurate depth estimation: an efficient semi-supervised deep neural network approach, in 2018 IEEE/CVF Conference on Computer Vision and Pattern Recognition Workshops (CVPRW), 18-22 June 2018, Salt Lake City, UT, USA, 1120-1127.

https://doi.org/10.1109/CVPRW.2018.00147

Surblys, V.; Ślaski, G.; Pikosz, H. 2018. The usage of a laser height sensors for estimating road unevenness profile, The Archives of Automotive Engineering - Archiwum Motoryzacji 79(1): 95-106.

Tettamanti, T.; Varga, I.; Szalay, Z. 2016. Impacts of autonomous cars from a traffic engineering perspective, Periodica Polytechnica Transportation Engineering 44(4): 244-250.

https://doi.org/10.3311/PPtr.9464

Udacity. 2016. Self-Driving Car: Datasets. GitHub, Inc. Available from Internet: https://github.com/udacity/self-driving-car/ tree/master/datasets

Van der Merwe, N. A.; Els, P. S.; Žuraulis, V. 2018. ABS braking on rough terrain, Journal of Terramechanics 80: 49-57. https://doi.org/10.1016/j.jterra.2018.10.003

Van Hamme, D.; Veelaert, P.; Philips, W. 2013. Lane identification based on robust visual odometry, in 16th International IEEE Conference on Intelligent Transportation Systems (ITSC 2013), 6-9 October 2013, The Hague, The Netherlands, 11791183. https://doi.org/10.1109/ITSC.2013.6728392

Więckowski, D.; Dąbrowski, K.; Ślaski, G. 2018. Adjustable shock absorber characteristics testing and modelling, IOP Conference Series: Materials Science and Engineering 421: 022039. https://doi.org/10.1088/1757-899X/421/2/022039

Wong, J. Y. 2008. Theory of Ground Vehicles. Wiley, 592 p.

Yang, Z.; Zhang, Y.; Yu, J.; Cai, J.; Luo, J. 2018. End-to-end multi-modal multi-task vehicle control for self-driving cars with visual perceptions, in 2018 24th International Conference on Pattern Recognition (ICPR), 20-24 August 2018, Beijing, China, 2289-2294. https://doi.org/10.1109/ICPR.2018.8546189 
Yi, J.; Alvarez, L.; Horowitz, R. 2002. Adaptive emergency braking control with underestimation of friction coefficient, IEEE Transactions on Control Systems Technology 10(3): 381-392. https://doi.org/10.1109/87.998027

ZF Friedrichshafen AG. 2011. CDC - Continuous Damping Control, ZF Friedrichshafen AG. Available from Internet: https://www.zf.com/products/en/cars/products_29310.html

Zhang, L.; Wu, E.-Y. 2009. A road segmentation and road type identification approach based on new-type histogram calculation, in 2009 2nd International Congress on Image and Signal Processing, 17-19 October 2009, Tianjin, China, 1-5. https://doi.org/10.1109/CISP.2009.5300878

Zhang, X.; Xu, Y.; Pan, M.; Ren, F. 2014. A vehicle ABS adaptive sliding-mode control algorithm based on the vehicle velocity estimation and tyre/road friction coefficient estimations, Vehicle System Dynamics: International Journal of Vehicle Mechanics and Mobility 52(4): 475-503. https://doi.org/10.1080/00423114.2013.864775

Zheng, T.; Wang, L.; Ma, F. 2011. Research on road identification method in anti-lock braking system, Procedia Engineering 15: 194-198. https://doi.org/10.1016/j.proeng.2011.08.039

Žuraulis, V.; Garbinčius, G.; Skačkauskas, P.; Prentkovskis, O. 2018. Experimental study of winter tyre usage according to tread depth and temperature in vehicle braking performance, Iranian Journal of Science and Technology, Transactions of Mechanical Engineering (in Press).

https://doi.org/10.1007/s40997-018-0243-0 